\title{
Choosing the Criteria for Clinical Evaluation of Composite Restorations: An Analysis of Impact on Reliabilty and Treatment Decision
}

Cleber Paradzinski Cavalheiro ${ }^{1}$, Pablo Soares de Souza ${ }^{1}$, Rachel de Oliveira Rocha² ${ }^{\circledR}$, Fausto

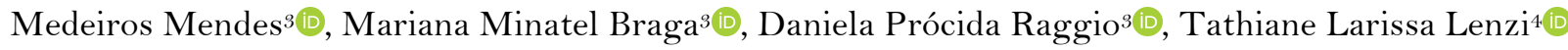

${ }^{1}$ Course of Dentistry, Federal University of Santa Maria, Santa Maria, RS, Brazil.

${ }^{2}$ Department of Stomatology, Federal University of Santa Maria, Santa Maria, RS, Brazil.

${ }^{3}$ Department of Orthodontics and Pediatric Dentistry, School of Dentistry, University of Sao Paulo, São Paulo, SP, Brazil.

${ }^{4}$ Post-Graduate Program in Pediatric Dentistry, School of Dentistry, Federal University of Rio Grande do Sul, Porto Alegre, RS, Brazil.

Author to whom correspondence should be addressed: Tathiane L. Lenzi, School of Dentistry, Post-Graduate Program in Pediatric Dentistry, Federal University of Rio Grande do Sul, Ramiro Barcelos 2492, Santa Cecília, Porto Alegre, RS, Brazil. 90035-003. Phone: +55 51 3308-5493. E-mail: tathilenzi@hotmail.com.

Academic Editors: Alessandro Leite Cavalcanti and Wilton Wilney Nascimento Padilha

Received: 14 June 2019 / Accepted: 21 February 2020 / Published: 24 March 2020

How to cite this article: Cavalheiro CP, Souza PS, Rocha RO, Mendes FM, Braga MM, Raggio DP, et al. Choosing the criteria for clinical evaluation of composite restorations: an analysis of impact on reliabilty and treatment decision. Pesqui Bras Odontopediatria Clín Integr. 2020; 20:e5088. https://doi.org/10.1590/pboci.2020.055

\begin{abstract}
Objective: To assess the reproducibility of two clinical criteria for the evaluation of restorations in primary teeth and the impact on treatment decision. Material and Methods: A cross-sectional study was performed selecting 71 resin-based composite restorations placed in primary molars of children who had sought dental treatment at a dental school. Two trained examiners evaluated independently the restorations using modified FDI and USPHS criteria. All restorations were assessed separately with each system in random order to avoid memory bias. Kappa statistics were used to determine inter-examiner reliability considering each parameter of both criteria and score final about treatment decision. McNemar test was used to compare the treatment decision with two criteria. The significance level was set at $5 \%$. Results: Kappa values ranged from 0.28 to 0.93 with USPHS and 0.28 to 0.88 with FDI, considering each parameter separately. Interexaminer agreement for treatment decision was excellent for both criteria (Kappa: 0.85-0.90). For clinical decision-making, no difference between criteria was found, irrespective of examiner. Conclusion: Low interexaminer agreement for evaluation of each parameter of USPHS and FDI criteria does not reflect on reproducibility for treatment decision. Both criteria may be suitable for evaluation of composite restorations in primary teeth.
\end{abstract}

Keywords: Clinical Decision-Making; Dental Restoration Failure; Tooth; Deciduous. 


\section{Introduction}

Resin-based dental composites are widely used in Pediatric Clinic for restoring anterior and posterior teeth. The annual failure rates of composite restorations in primary teeth have varied between $4 \%$ and $9 \%$ [1,2]. Nevertheless, parameters for assessing the restorations' quality are often subjective, where small deviations from ideal concepts determine the replacement.

In this sense, different criteria have been proposed aiming to standardize the evaluation of restorative materials or operative techniques in clinical trials. Furthermore, these criteria may be useful for quality assessment of restorations placed by clinicians in their own practices. Also, dental students should be trained to use them as part of a clinical evaluation to determine whether a restoration can be maintained or whether it needs repair or replacement [3].

US Public Health Service (USPHS) guidelines also known as the "Ryge criteria" [4] and FDI (World Dental Federation) [3] are the criteria most used for evaluating composite restorations [5-9]. Both criteria are based on assessment of biological, esthetic and functional parameters and can be and adjusted according to the needs of the user. FDI criteria were recently proposed as "standard criteria", more sensitive for identifying differences in dental restorations [3].

Good criteria should be reproducible. However, no previous study has compared the inter-examiner agreement when using the two criteria for clinical evaluation of restorations. Besides, criteria that lead to overtreatment would not be desirable nowadays. The impact of using these criteria in the treatment decision regarding the evaluated restorations was not yet investigated.

Therefore, we aimed to assess the reproducibility of two clinical criteria for the evaluation of resinbased composite restorations in primary teeth and the impact on treatment decision.

\section{Material and Methods}

Sample Selection

A convenience sample was used in this study. Seventy-one resin-based composite restorations placed in primary molars were selected from clinical records of patients attended at Pediatric Clinic of the School of Dentistry, Federal University of Santa Maria. Occlusal restorations were performed by fourth and five years dental undergraduate students, supervised by specialists in Pediatric Dentistry. The majority of the children have low familiar socioeconomic status and high caries risk.

Training and Calibration of Evaluators

Two examiners (C.P.C. and P.S.S.) underwent a total of $8 \mathrm{~h}$ of specific training session involving theoretical explanations and discussion using clinical slides about United States Public Health Service (USPHS) and World Dental Federation (FDI) criteria. The responsible for training session was a benchmark examiner (T.L.L.) who has been trained and calibrated for using two criteria. The examiners' calibration procedures considered two examinations of 20 photographs that were representative of each score for both criteria, randomly distributed in both periods, for Cohen’s Kappa calculation (Kappa = 0.80).

A modified USPHS guidelines was used in this study [10], including color match, marginal adaptation, anatomic form, marginal staining, surface roughness and caries. FDI criteria were categorized into three groups [3]: esthetic (four criteria), functional (three criteria) and biological (one criterion) parameters. A five-point Likert scale was used to assess the functional property "patient view" of FDI criteria in the Pediatric 
Dentistry. Child's satisfaction with the restoration was measured from one to five according to the scale: $1=$ very satisfied; 2 = satisfied; $3=$ indifferent; 4 = unsatisfied; 5 = very unsatisfied.

For both criteria, postoperative sensitivity was not considered because this evaluation is subject to subjectivity when performed in pediatric patients.

\section{Evaluation of Restorations}

The children were called to visit the dental clinic. After prophylaxis, the two trained examiners (C.P.C. and P.S.S.) evaluated independently the children's restorations using ballpoint probe and plane buccal mirror (Hu-Friedy Manufacturing Co., Chicago, USA). All restorations were assessed separately with each criterion and randomly distributed to avoid memory bias.

Each criterion of FDI can be expressed with five scores, three for acceptable (1. clinically very good; 2. clinically good; 3. clinically sufficient/satisfactory) and two for non-acceptable (4. clinically unsatisfactory repairable restoration; 5. clinically poor - restoration replacement). Codes Alfa, Bravo, Charlie and Delta were used to rate the restorations according to the assigned descriptive values for each characteristic of USPHS criteria. For clinical decision-making, the worst grading among all parameters of both criteria was considered. The restorations were recorded as failed if they were classified as Bravo for caries or Charlie and Delta scores for the other parameters using USPHS criteria or rated as scores 4 and 5 by FDI criteria.

\section{Statistical Analyses}

The descriptive analysis provides the distribution summary of restorations according to the parameters assessed with both criteria. Kappa values and respective 95\% confidence intervals were calculated and used to determine inter-examiner reliability considering each parameter of both criteria and score final about treatment decision. The strength of agreement for the Kappa value can be interpreted as follows: $<0.20$ : Poor; 0.21-0.40: Fair; 0.41-0.60: Moderate; 0.61-0.80: Good; 0.81-1.00: Excellent [11]. McNemar test was used to compare the treatment decision with two criteria. The significance level was set at $p<0.05$. Statistical analyses were performed using the STATA software 12.0 (Stata Corp., College Station, TX, USA).

\section{Ethical Concern}

The research protocol was approved by the Local Research Board and the parents or guardians signed a written informed consent. The personal information of the children was kept confidential.

\section{Results}

The distribution of restorations according to the parameters evaluated by two examiners using USPHS and FDI criteria is displayed in Tables 1 and 2, respectively.

Most restorations were classified as Alfa for all parameters of the USPHS criteria. Only surface roughness was categorized as Delta. The main reason for need of intervention was adjacent caries. Likewise, the majority of restorations were rated as score (clinically very good) for all parameters of the FDI criteria. However, there was not a main reason for need of intervention, being fracture, marginal adaptation and recurrence caries factors more related to indication of restorations' replacement or repair. Only one of examiners judged need of repair (score 4) due color and anatomic form. 
Table 1. Status of the restorations according to USPHS criteria.

\begin{tabular}{|c|c|c|c|c|c|c|c|c|}
\hline \multirow{5}{*}{ USPHS Criteria } & \multicolumn{8}{|c|}{ Examiner } \\
\hline & $\mathrm{A}$ & B & A & B & $\mathrm{A}$ & B & $\mathrm{A}$ & $\mathrm{B}$ \\
\hline & \multirow{2}{*}{\multicolumn{2}{|c|}{ Alfa }} & \multicolumn{2}{|c|}{ Bravo } & \multicolumn{2}{|c|}{ Charlie } & \multicolumn{2}{|c|}{ Delta } \\
\hline & & & \multicolumn{6}{|c|}{ Restorations } \\
\hline & $\mathrm{N}(\%)$ & $\mathrm{N}(\%)$ & $\mathrm{N}(\%)$ & $\mathrm{N}(\%)$ & $\mathrm{N}(\%)$ & $\mathrm{N}(\%)$ & $\mathrm{N}(\%)$ & $\mathrm{N}(\%)$ \\
\hline Color Match & $55(77.5)$ & $61(86.0)$ & $13(18.3)$ & $7(9.8)$ & $3(4.2)$ & $3(4.2)$ & - & - \\
\hline Marginal Adaptation & $55(77.5)$ & $62(87.4)$ & $10(14.1)$ & $3(4.2)$ & $6(8.4)$ & $6(8.4)$ & - & - \\
\hline Anatomic Form & $54(76.1)$ & $60(84.6)$ & $12(16.9)$ & $6(8.4)$ & $5(7.0)$ & $5(7.0)$ & - & - \\
\hline Surface Roughness & $55(77.5)$ & $57(80.4)$ & $10(14.1)$ & $9(12.6)$ & - & - & $6(8.4)$ & $5(7.0)$ \\
\hline Marginal Pigmentation & $50(70.4)$ & $55(77.5)$ & $18(25.4)$ & $12(16.9)$ & $3(4.2)$ & $4(5.6)$ & - & - \\
\hline Occlusal Contact & $63(88.8)$ & $64(90.1)$ & $3(4.2)$ & $2(2.9)$ & $5(7.0)$ & $5(7.0)$ & - & - \\
\hline Adjacent Caries & $63(88.8)$ & $61(85.9)$ & $8(11.2)$ & $10(14.1)$ & - & - & - & - \\
\hline
\end{tabular}

Table 2. Status of the restorations according to FDI criteria.

\begin{tabular}{|c|c|c|c|c|c|c|c|c|c|c|}
\hline \multirow{5}{*}{ FDI Criteria } & \multicolumn{10}{|c|}{ Examiner } \\
\hline & A & B & A & B & A & B & $\mathrm{A}$ & $\mathrm{B}$ & A & B \\
\hline & \multicolumn{2}{|c|}{1} & \multicolumn{2}{|c|}{2} & \multicolumn{2}{|c|}{3} & \multicolumn{2}{|c|}{4} & \multicolumn{2}{|c|}{5} \\
\hline & \multicolumn{10}{|c|}{ Restorations } \\
\hline & $\mathrm{N}(\%)$ & $\mathrm{N}(\%)$ & $\mathrm{N}(\%)$ & $\mathrm{N}(\%)$ & $\mathrm{N}(\%)$ & $\mathrm{N}(\%)$ & $\mathrm{N}(\%)$ & $\mathrm{N}(\%)$ & $\mathrm{N}(\%)$ & $\mathrm{N}(\%)$ \\
\hline Surface Luster & $59(83.1)$ & $56(78.9)$ & $10(14.1)$ & $13(18.3)$ & $1(1.4)$ & $1(1.4)$ & - & - & $1(1.4)$ & $1(1.4)$ \\
\hline Surface Staining & $58(81.6)$ & $62(87.3)$ & $10(14.1)$ & $6(8.4)$ & $1(1.4)$ & $1(1.4)$ & - & - & $2(2.9)$ & $2(2.9)$ \\
\hline Margin Staining & $39(54.9)$ & $44(62.0)$ & $24(33.8)$ & $23(32.2)$ & $6(8.4)$ & $2(2.9)$ & - & - & $2(2.9)$ & $2(2.9)$ \\
\hline Color Match and Translucency & $52(73.2)$ & $62(87.3)$ & $10(14.1)$ & $4(5.6)$ & $6(8.4)$ & $3(4.2)$ & $1(1.4)$ & - & $2(2.9)$ & $2(2.9)$ \\
\hline Anatomic Form & $51(71.7)$ & $59(83.1)$ & $11(15.5)$ & $7(9.8)$ & $5(7.0)$ & $3(4.2)$ & $2(2.9)$ & - & $2(2.9)$ & $2(2.9)$ \\
\hline Fracture & $62(87.3)$ & $61(86.0)$ & $1(1.4)$ & $1(1.4)$ & - & - & $2(2.9)$ & $4(5.6)$ & $6(8.4)$ & $5(7.0)$ \\
\hline Marginal Adaptation & $51(71.8)$ & $57(80.4)$ & $9(12.7)$ & $5(7.0)$ & $4(5.6)$ & $5(7.0)$ & $2(2.9)$ & $1(1.4)$ & $5(7.0)$ & $3(4.2)$ \\
\hline Patient View* & $50(70.4)$ & $54(76.1)$ & $17(24.0)$ & $11(15.5)$ & $3(4.2)$ & $5(7.0)$ & $1(1.4)$ & $1(1.4)$ & - & - \\
\hline Caries Recurrence & $57(80.4)$ & $61(85.9)$ & $5(7.0)$ & $3(4.2)$ & $1(1.4)$ & $1(1.4)$ & $5(7.0)$ & $4(5.6)$ & $3(4.2)$ & $2(2.9)$ \\
\hline
\end{tabular}

*Adapted for Pediatric Dentistry by five-point Likert scale. 
Tables 3 and 4 present unweighted Kappa coefficient for inter-examiner reproducibility for USPHS and FDI, respectively. Overall, lower Kappa values were obtained with FDI criteria. When using USPHS criteria, Kappa values ranged from 0.28 to 0.93 considering each parameter separately. Fair agreement was achieved only for marginal pigmentation assessment. Inter-examiner agreement was good for adjacent caries, excellent for occlusal contact and moderate for other parameters.

Table 3. Unweighted Kappa coefficient for inter-examiner reproducibility with USPHS criteria.

\begin{tabular}{lccc}
\hline \multicolumn{1}{c}{ USPHS Criteria } & Kappa & SE & 95\% CI \\
\hline Color Match & 0.55 & 0.12 & $0.31-0.80$ \\
Marginal Adaptation & 0.50 & 0.12 & $0.27-0.73$ \\
Anatomic Form & 0.50 & 0.12 & $0.26-0.74$ \\
Surface Roughness & 0.48 & 0.12 & $0.25-0.72$ \\
Marginal Pigmentation & 0.28 & 0.12 & $0.03-0.52$ \\
Occlusal Contact & 0.93 & 0.07 & $0.78-1.00$ \\
Adjacent Caries & 0.75 & 0.12 & $0.51-0.98$ \\
Treatment Decision & 0.85 & 0.08 & $0.69-1.00$ \\
\hline
\end{tabular}

Table 4. Unweighted Kappa coefficient for inter-examiner reproducibility with FDI criteria.

\begin{tabular}{lccc}
\hline \multicolumn{1}{c}{ FDI Criteria } & Kappa & SE & 95\% CI \\
\hline Surface Luster & 0.34 & 0.14 & $0.06-0.61$ \\
Surface Staining & 0.41 & 0.10 & $0.21-0.60$ \\
Margin Staining & 0.28 & 0.15 & $-0.01-0.57$ \\
Color Match and Translucency & 0.35 & 0.10 & $0.15-0.56$ \\
Anatomic Form & 0.38 & 0.11 & $0.16-0.59$ \\
Fracture & 0.88 & 0.08 & $0.73-1.00$ \\
Marginal Adaptation & 0.47 & 0.09 & $0.29-0.65$ \\
Patient View & 0.50 & 0.10 & $0.30-0.70$ \\
Caries Recurrence & 0.58 & 0.12 & $0.34-0.81$ \\
Treatment Decision & 0.90 & 0.07 & $0.76-1.00$ \\
\hline
\end{tabular}

When using FDI criteria, values ranged from 0.28 to 0.88. Excellent agreement was obtained only for fracture evaluation. For other parameters, Kappa values were interpreted as poor or fair. Although the interexaminer agreement was fair considering the global score (the worst grading among all parameters), excellent agreement was achieved for treatment decision.

Kappa values for treatment decision were 0.85 and 0.90 with USHPS and FDI criteria. For clinical decision-making, no difference between criteria was found, irrespective of examiner (Table 5).

Table 5. Comparison of the treatment decision according to two criteria.

\begin{tabular}{lcccccc}
\hline Treatment Decision & \multicolumn{3}{c}{ Examiner A } & \multicolumn{3}{c}{ Examiner B } \\
& USPHS & FDI & p-value & USPHS & FDI & p-value \\
\hline No Intervention & $59(83.1)$ & $60(84.5)$ & 1.000 & $58(81.7)$ & $58(81.7)$ & 1.000 \\
Intervention & $12(16.9)$ & $11(14.5)$ & & $13(18.3)$ & $13(18.3)$ & \\
\hline
\end{tabular}

\section{Discussion}

This study provides valuable information regarding the reliability and clinical decision-making of two criteria commonly used to evaluate restorations' quality. Although low Kappa values have been obtained considering the assessment of each parameter for both criteria, this fact did not impact the treatment decision, 
as the reliability was good. This finding is important because the final objective of a diagnostic method is to reach a consistent treatment decision.

Most restorations were classified as clinically acceptable. FDI criteria are categorized in five scores, being three acceptable. The differences among them, mainly between scores 1 and 2 , are subtle and more prone to disagrements, as observed in this study (Table 2). On the other hand, the characteristics related to Alpha and Bravo scores of USPHS are less subjective.

Unweighted Kappa was used in this study. Although it was expected high values with weighted Kappa, we aimed to assess if low values for each parameter, including all categories of the criteria, would result on low inter-examiner agreement for treatment decision. For clinical decision-making, the most severe grading among all parameters of both criteria prevailed. Despite the fair inter-agreement for majority of the parameters, excelent reliably was found for treatment decision. Including, the Kappa values of global restorations' evaluation was slightly higher with FDI than USPHS. Based on these findings, a simplified clinical evaluation, mainly when using FDI, may be appropriate, e.g. it is possible to pool scores 1 and 2 (equivalent to USPHS score A), resulting in four different scores (two acceptable and two unacceptable), or even to combine scores 1,2 and 3 to only one acceptable score and additionally two or one (merged scores 4 and 5) unacceptable score [3].

For clinical decision-making, no statistically significant difference between criteria was found, irrespective of the examiner. We hypothesized that differences would be noted if a high proportion of insatifactory restorations were included in the sample. Whenever a restoration receives a score of 4 or 5 of FDI or Bravo for caries and Charlie or Delta scores of USPHS, it must be judged with need of intervention. However, differently of USPHS guidelines, FDI criteria allow deciding whether the restoration can be repaired (score 4) or require replacement (score 5). In this sense, decision-making might differ between two criteria, since FDI could avoid a more invasive intervention or even overtreatment. Repairing is a interesting approach that saves patient-chair time and tooth structure [12,13], being less likely to need an aggressive treatment, as endodontic treatment or extraction [14].

Secondary caries and marginal defects are the most frequent reasons for replacement reported in the literature [15]. Similar Kappa values between criteria were achieved for marginal adaptation assessment. Inter-examiner agreement was good (0.75) and moderate (0.58) for USPHS and FDI. It is relevant to highlight that USPHS rates caries as present or absent, while FDI involves all stages of carious process, i.e., since initial demineralization until deep caries (accessible or not for repair). Again, the main inter-examiner disagreements were between FDI scores 1 (no caries) and 2 (small and localized demineralization). In this study, a mean of $12.6 \%$ and $9.8 \%$ of restorations were judged as failure due caries with USPHS and FDI criteria, respectively. However, as treatment decision, only a mean of $3.5 \%$ of restorations needed to be replaced due caries when FDI was used.

The small number of evaluators can be considered a limitation of the study, as only two examiners were used. Conversely, a larger number of examiners would imply a more lengthy assessment for each child, making it more tiring and stressful for the young children who participated in this study. Moreover, it would have increased the number of sessions, which could have led to dropouts. For this reason, intra-examiner reprodutibily was not calculated in this study. It should be noted that a good intra-examiner agreement was achevied during the training and calibration stage.

In this sense, low Kappa values for the evaluation of some parameters seem to be more related a limitation of the criteria and not of the evaluators' training. Among evaluated parameters, lowest Kappa values 
were obtained for marginal pigmentation when using both criteria. Even using a criterion, this parameter was suitable to higher subjectivity. Marginal pigmentation can occur due degradation of bonding agents or penetration of dyes from dietetic habits; factors not directly associated to caries. In this sense, this evaluation migh to have low impact in restorations' quality of posterior teeth.

The property "patient view" of FDI criteria was measured with a five point Likert scale. Although it is a validated instrument [16], the responses of the children differed in two examinations (Kappa equal 0.50). This indicates that child's satisfaction may be not related to real condition of restorations and researches should rethink whether this evaluation is necessary in future studies.

\section{Conclusion}

Low inter-examiner agreement for evaluation of each parameter of USPHS and FDI criteria does not reflect on reproducibility for treatment decision. Both criteria may be suitable for evaluation of composite restorations in primary teeth. Other aspects should be considered for choosing the clinical criteria, such as time consuming and examiner preferences.

\section{Authors' Contributions}

\begin{tabular}{|c|c|c|}
\hline $\mathrm{CPC}$ & (iD) $0000-0001-9402-1811$ & Investigation, Formal Analysis and Writing - Original Draft Preparation. \\
\hline PSS & (iD) $0000-0001-7829-4035$ & Investigation, Formal Analysis and Writing - Original Draft Preparation. \\
\hline ROR & (iD) $0000-0001-7737-2257$ & Writing - Original Draft Preparation and Writing - Review and Editing. \\
\hline FMM & (iD) $0000-0003-1711-4103$ & Validation, Formal Analysis and Writing - Review and Editing. \\
\hline MMB & (iD) $0000-0002-4469-6500$ & Writing - Original Draft Preparation and Writing - Review and Editing. \\
\hline DPR & (iD) $0000-0002-0048-2068$ & Writing - Original Draft Preparation and Writing - Review and Editing. \\
\hline TLL & (iD) $0000-0003-3568-5217$ & Conceptualization, Methodology, Investigation and Writing - Review and Editing. \\
\hline
\end{tabular}

\section{Financial Support}

The Institutional Program of Scientific Initiation Grants of the Brazilian National Council for Scientific and Technological Development $(\mathrm{CNPq})-\mathrm{PIBIC} / \mathrm{CNPq}$.

\section{Conflict of Interest}

The authors declare no conflicts of interest.

\section{References}

[1] Bücher K, Tautz A, Hickel R, Kühnisch J. Longevity of composite restorations in patients with early childhood caries (ECC). Clin Oral Investig 2014; 18(3):775-82. https://doi.org/10.1007/s00784-013-1043-y

[2] Pinto GS, Oliveira LJ, Romano AR, Schardosim LR, Bonow ML, Pacce M, et al. Longevity of posterior restorations in primary teeth: results from a paediatric dental clinic. J Dent 2014; 42(10):1248-54. https://doi.org/10.1016/j.jdent.2014.08.005

[3] Hickel R, Peschke A, Tyas M, Mjör I, Bayne S, Peters M, et al. FDI World Dental Federation - clinical criteria for the evaluation of direct and indirect restorations. Update and clinical examples. J Adhes Dent 2010; 12(4):259-72. https://doi.org/10.3290/j.jad.a19262

[4] Cvar JF, Ryge G. Reprint of criteria for the clinical evaluation of dental restorative materials. Clin Oral Investig 2005; 9(4):2 15-32. https://doi.org/10.1007/s00784-005-0018-Z

[5] dos Santos MP, Passos M, Luiz RR, Maia LC. A randomized trial of resin-based restorations in class I and class II beveled preparations in primary molars: 24-month results. J Am Dent Assoc 2009; 140(2):156-66. https://doi.org/10.14219/jada.archive.2009.0129

[6] Casagrande L, Dalpian DM, Ardenghi TM, Zanatta FB, Balbinot CE, García-Godoy F, et al. Randomized clinical trial of adhesive restorations in primary molars. 18-month results. Am J Dent 2013; 26(6):351-5. 
[7] Sengul F, Gurbuz T. Clinical evaluation of restorative materials in primary teeth Class II lesions. J Clin Pediatr Dent 2015; 39(4):315-21. https://doi.org/10.17796/1053-4628-39.4.315

[8] Bektas Donmez S, Uysal S, Dolgun A, Turgut MD. Clinical performance of aesthetic restorative materials in primary teeth according to the FDI criteria. Eur J Paediatr Dent 2016; 17(3):202-12.

[9] Pascon FM, Kantovitz KR, Caldo-Teixeira AS, Borges AF, Silva TN, Puppin-Rontani RM, et al . Clinical evaluation of composite and compomer restorations in primary teeth: 24-month results. J Dent 2006; 34(6):381-8. https://doi.org/10.1016/j.jdent.2005.08.003

[10] Wilson MA, Cowan AJ, Randall RC, Crisp RJ, Wilson NH. A practice-based, randomized, controlled clinical trial of a new resin composite restorative: one-year results. Oper Dent 2002; 27(5):423-9.

[11] Foley JI. Dental students consistency in applying the ICDAS system within paediatric dentistry. Eur Arch Paediatr Dent 2012; 13(6):319-22. https://doi.org/10.1007/bfo3320834

[12] Moncada G, Vildósola P, Fernández E, Estay J, de Oliveira Júnior OB, de Andrade MF, et al. Longitudinal results of a 10-year clinical trial of repair of amalgam restorations. Oper Dent 2015; 40(1):34-43.

https://doi.org/10.2341/14-045-C

[13] Gordan VV, Riley JL 3rd, Blaser PK, Mondragon E, Garvan CW, Mjör IA. Alternative treatments to replacement of defective amalgam restorations: results of a seven-year clinical study. J Am Dent Assoc 2011; 142(7):842-9. https://doi.org/10.14219/jada.archive.2011.0274

[14] Gordan VV, Riley J 3rd, Geraldeli S, Williams OD, Spoto JC 3rd, Gilbert GH, et al. The decision to repair and replace a defective restoration is affected by who placed the original restoration: findings from the National Dental PBNR. J Dent 2014; 42(12):1528-34. https://doi.org/10.1016/j.jdent.2014.09.005

[15] Deligeorgi V, Mjör IA, Wilson NH. An overview of reasons for the placement and replacement of restorations. Prim Dent Care 2001; 8(1):5-11. https://doi.org/10.1308/135576101771799335

[16] Wong DL, Baker CM. Pain in children: comparison of assessment scales. Pediatr Nurs 1988; 14(1):9-17. 